
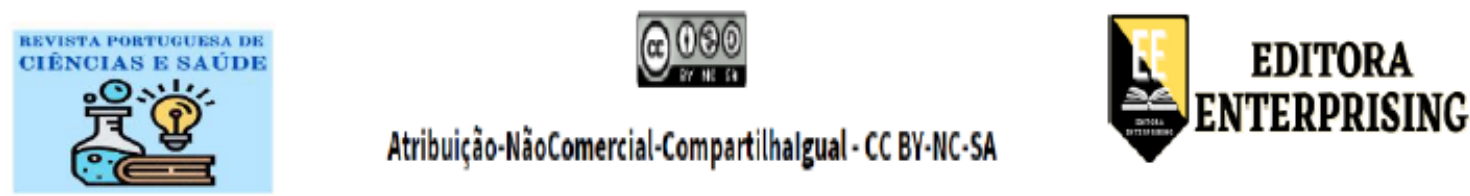

\title{
Unidade de Terapia Intensiva-Pediátrica: Compreensão das Necessidades dos Familiares dos Pacientes Internados
}

\author{
Anne Caroline Cavalcante da Rocha ${ }^{1}$ \\ Marcella Lima Marinho ${ }^{2}$ \\ Helenira Macedo Barros Machado ${ }^{3}$ \\ Marco José Mendonça de Souza ${ }^{4}$ \\ Cleiry Simone Moreira da Silva ${ }^{5}$
}

\begin{abstract}
RESUMO
A internação de um paciente em Unidades de Terapia Intensiva Pediátrica (UTI- P), ocorre geralmente de forma aguda e inesperada, restando pouco tempo para o ajustamento e compreensão dos fatores sociopatológicos pelos membros da família. Diante dessa situação, os familiares podem sentir-se desamparados e com dificuldades para se mobilizarem, fazendo emergir diferentes tipos de necessidades que podem ser agravadas pela falta de informação e conhecimento prévio em relação ao ambiente das UTI. Objetivo: Averiguar sobre os desafios dos familiares de pacientes que se encontram hospitalizados na Unidade de Terapia Intensiva Pediátrica (UTIP). Método: trata-se de uma revisão integrativa que busca compreender as dificuldades dos familiares de pacientes internados na UTI-P, e entender como é feito o acolhimento à essas famílias. O trabalho ocorreu mediante a uma pesquisa literária que contemplou as pesquisas realizadas com bases em artigos científicos, disponíveis nos bancos de dados: Scielo e Lilacs, no período de 2018 a 2021 Resultado: demonstram o quão importante é o acolhimento a família do paciente internado na UTIP, e a eficácia da equipe ao ver a família como um todo no processo biopsíquico-social do tratamento da criança que se encontra acometido na UTI-P.
\end{abstract}

Palavra-chave: Acolhimento, Unidade de Terapia Intensiva Pediátrica, família.

\footnotetext{
${ }^{1}$ Discente do Curso em Bacharelado em Enfermagem do Centro Universitário Estácio da Amazônia, e-mail: juliannegarcia83@gmail.com

${ }^{2}$ Docente do Curso em Bacharelado em Enfermagem do Cento Universitário Estácio da Amazônia, e-mail: helenira.barros@estacio.br

${ }^{3}$ Docente do Curso em Bacharelado em Enfermagem do Centro Universitário Estácio da Amazônia, e-mail: marcela.lima@estacio.com.

${ }^{4}$ Docente do Curso IFRR

Docente do Curso Superior de Licenciatura em Educação Física do Instituto federal de Roraima, e-mail: Marco.souza@ifrr.edu.br

${ }^{4}$ Docente do Curso em Bacharelado em Enfermagem do Centro Universitário Estácio da Amazônia, e-mail: cleiry.silva@estacio.br
}

INTRODUÇÃO

Ter uma criança hospitalizada em uma Unidade de Terapia Intensiva Pediátrica (UTIP), na visão dos familiares é um momento de extrema angústia e medo, por ser um ambiente complexo tornando-se assim ameaçador, despertando um sentimento de impotência. Então a comunicação entre família e equipe de enfermagem é um dos pontos de importância para uma

Rocha, A.C.C., Marinho, M.L., Machado, H.M.B., Souza, M.J.M., Silva, C.S.M.; Unidade de Terapia IntensivaPediátrica: Compreensão das Necessidades dos Familiares dos Pacientes Internados. Revista Portuguesa de Ciências e Saúde V.2, Nº1, p.60-76, Jan/Jul. 2020. Artigo recebido em 25/06/2021. Última versão recebida em 10/07/2021. Aprovado em 20/08/2021. 
Unidade de Terapia Intensiva-Pediátrica: Compreensão das Necessidades dos Familiares dos Pacientes Internados

boa relação entre ambos. $\mathrm{O}$ acolhimento adequado nessa situação de estresse tem um papel fundamental (NASCIMENTO, 2021).

A UTI-P é uma unidade que reúne médicos, enfermeiros, técnicos e auxiliares de enfermagem que estão ali para auxiliar e prestar serviços ao paciente crítico, no enfoque de reduzir o nível de mortalidade através dos cuidados intensivos. Sensibilizar a família sobre esses cuidados pode trazer uma percepção diferente de cuidado. A presença de um familiar promove e mantém a inter-relação da criança com a família, neutralizando os efeitos decorrentes da separação, melhorando sua adaptação no hospital e trazendo um pouco mais de tranquilidade de ambas as partes (MOLINA, 2009).

As necessidades de familiares são conceituadas como algo essencial, exigido pela pessoa que quando suprido, alivia ou diminui a aflição e angústia imediata ou melhora a percepção e adequação de bem-estar. As necessidades não atendidas ou inadequadamente atendidas trazem desconforto e períodos de desequilíbrio, que podem levar o ser humano a precisar de auxílio de profissional habilitado, por isso é muito importante essa preocupação, esse olhar humanizado não só com o paciente, mas também com os familiares (SOUSA, 2014).

Nas últimas duas décadas, tem havido um movimento de se recriar e redescobrir como a família possa ser envolvida na prática de enfermagem. Trazer o foco para a família no cuidado, aborda novas teorias no sentindo de proporcionar a compreensão à enfermagem e a família. A enfermagem no contexto do cuidado da saúde e da doença, enfatizando os problemas atuais de saúde que a família esteja vivenciando (BOUSSO,2001).

Segundo Trevelbee (1979) foca as relações interpessoais com o objetivo de auxiliar o indivíduo e a família a enfrentar a doença e o sofrimento propondo um cuidar holístico é visar o paciente e a família como um ser humano que naquele momento precisa de assistência humanizada que envolve empatia. A família precisa enxergar o cuidado que a equipe de enfermagem presta com o paciente, a visualização traz a sensação de controle com o que está acontecendo. Ao passar dos anos o acolhimento à família do paciente vem criando um pouco mais de força no ambiente hospitalar, porém, especificando a UTI precisa de um enfoque maior, por ser uma unidade de alta complexidade isso pode passar despercebido.

Nesses ambientes críticos com a prática mecanizada e especializada, é preciso refletir sobre o sentido real da assistência como uma forma de cuidado (RODRIGUES, 2016). A percepção da família no cuidado ao paciente internado na UTIP é definida através da orientação que é passada para ela pela equipe de enfermagem, atualmente as ferramentas utilizadas para a 
Unidade de Terapia Intensiva-Pediátrica: Compreensão das Necessidades dos Familiares dos Pacientes Internados

informação é a comunicação direta da equipe para a família isso desperta um cuidado e empatia daquele profissional e a família do paciente percebi a preparação da equipe.

O programa de humanização da assistência hospitalar (2001) assegura o direito da família sobre o acolhimento humanizado dada pela equipe de enfermagem. Construir um ambiente de conforto e confiança é necessário para se obter uma relação favorável entre a equipe e os usuários.

Segundo Sousa (2014) a enfermagem sempre reconheceu a importância da família na promoção e manutenção da saúde tendo-a como unidade do cuidado com ênfase nas suas respostas aos problemas atuais e potenciais de saúde que esteja vivenciando assim como aos significados construídos simbolicamente ao adoecimento. Desse modo as necessidades dos familiares são identificadas e atendidas.

Segundo Freire (2008) nesse contexto, o acolhimento é uma das estratégias de mudanças do processo de trabalho em saúde. Essa estratégia busca alterar as relações entre profissionais e usuários. Assim, estabelecendo um vínculo e a humanização no acolhimento familiar.

Compreendendo que a hospitalização da criança em UTIP é altamente estressante, afeta de inúmeras maneiras todos os membros da família que tem participação no cuidado diário da criança e no cotidiano familiar; torna-se relevante o estudo; pois a UTI-P apresenta um ambiente de fortes emoções, conflitos e sentimentos, que engloba desde as crianças e pais até a equipe de saúde. Através de vertentes diferentes, cada um apresenta uma vulnerabilidade que deve ser atendida, pois isso significa humanização, a preocupação e atenção com o outro (RAAD, 2006).

O modelo de atenção à saúde direcionado à criança e aos seus respectivos responsáveis surge como uma tentativa de transformação de realidade vivida em uma hospitalização infantil. Os profissionais de saúde que optam pela assistência humanizada, devem buscar compreender a importância da relação pais-filhos, e incentivar o relacionamento dos pais com a criança durante a hospitalização afim de consolidar os laços de afinidade entre ambos, pois essa aproximação é muito importante tanto para a criança-paciente como para o familiar (COSTA, 2001).

São pressupostos para a efetivação do cuidado humanizado as práticas de saúde que respeitam a individualidade do ser humano e que contemplem todas as suas dimensões biopsicossocial, cultural e espiritual. As ações da equipe de enfermagem que observam os princípios bioéticos são fundamentadas no bom relacionamento humano com educação e empatia. Quando você pergunta às pessoas o que elas entendem sobre atendimento humanizado, 
Unidade de Terapia Intensiva-Pediátrica: Compreensão das Necessidades dos Familiares dos Pacientes Internados

elas associam a como os profissionais tratam o paciente e os familiares, com educação e gentileza (AMARAL, 2016).

O grande desafio hoje a ser enfrentado pelos profissionais nas UTI é a humanização da assistência, já que a tecnologia cada vez mais se supera e, muitas vezes, verificamos que estamos envolvidos com as máquinas, ansiosos e atentos ao que elas mostram, esquecendo de que estamos cuidando de pessoas, que o olhar clínico naquele paciente e naquele familiar é bem mais importante (BARBOSA, 2004).

De acordo com Nogare (2001), não há humanismo sem autonomia. Isto é, a humanização conduz o crescimento, à liberdade de criação e de vida, estabelece as possibilidades e reciprocidade entre homens em nível individual e grupal com relação aos direitos e deveres e condições do bem-estar e bem viver, ou seja, a humanização traz benefícios não só para o paciente e o familiar, mas também para a equipe de saúde.

O processo de humanização envolve mais que permitir ou não a presença dos pais na UTI, é importante que a enfermagem seja um elemento de confiança à família, já que é ela quem deve ajudá-la a passar por essa experiência. Com o atendimento humanizado à família, essa preocupação a mais não apenas com a criança, mas também com os pais, faz com que a equipe de enfermagem galgue a confiança deles, e uma relação de confiança entre a equipe de saúde e os familiares é muito importante para o tratamento da criança (BOUSSO, 2001).

De acordo com os autores Cardoso (2013), afirmam que o cuidar centrado na família é imprescindível no processo de assistência integral e humanizada em saúde e deve considerar cada núcleo, membro e dinâmica familiar em sua singularidade, pois o tratamento de cada família tem suas particularidades de acordo com suas necessidades.

A internação no filho na UTI induz os genitores a vivenciar angústias, aflições e medos. Ao enfrentar esta situação os pais colocam em jogo seus sentimentos e emoções que se confrontam com o caráter que assume na família e na sociedade, neste turbilhão de emoções esperam dar sequência ao seu ser no mundo, tentando conciliar o que sente com o que pensam e com o que tem de fazer (BARROS, 2017).

$\mathrm{O}$ atendimento humanizado da equipe de enfermagem aos familiares de pacientes de Unidade de Terapia Intensiva Pediátrica é muito importante como já foi dito, pois gera não só benefícios para a criança pois contribui para sua melhor recuperação, mas também para a relação entre a equipe e a família, incluindo confiança, assim o profissional tem um melhor desempenho 
Unidade de Terapia Intensiva-Pediátrica: Compreensão das Necessidades dos Familiares dos Pacientes Internados

em exercer seu trabalho, e o familiar tem menos medo de que o pior aconteça, e passa ter mais esperança de cura da criança.

No cenário, que a família e a equipe ocupam na UTI-P, a realização de ações ou ferramentas educativas para a melhor compreensão dos familiares, acerca do processo clínico e assistencialista ao paciente, é de grande valia para o conhecimento dos mesmos. Contudo, essa estratégia pode auxiliar a equipe no acolhimento humanizado, para uma boa relação de confiança e segurança nos procedimentos que são realizados com o paciente pediátrico. A família deve ser acolhida com base no acolhimento científico e psicológico sobre os cuidados a criança internada.

A pesquisa tem como foco compreender as necessidades dos familiares do paciente buscando um acolhimento eficaz, assim estabelecendo uma comunicação ampla, entre equipe de enfermagem e os familiares, com embasamento nós estudos científicos para melhor compreensão da percepção familiar.

Portanto, a pesquisa objetivou averiguar sobre os desafios dos familiares de pacientes que se encontra hospitalizado na Unidade de Terapia Intensiva Pediátrica. Assim, é importante que o enfermeiro, assim como qualquer outro profissional da saúde compreendam que a família assim como a criança hospitalizada, necessitam atenção e cuidados e que suas necessidades possam serem atendidas.

\section{PROCEDIMENTO METODOLÓGICO}

A pesquisa trata-se de uma revisão integrativa que busca compreender as dificuldades dos familiares de pacientes internados na Unidade de Terapia Intensiva Pediátrica, e entender como é feito o acolhimento à essas famílias. O processo metodológico iniciou com a busca artigos científicos, disponíveis em bancos de dados: SCIELO (Scientific Electronic Library Online) e LILACS (Literatura Latino-Americana do Caribe em Ciências da Saúde) foram utilizadas as seguintes combinações de palavras como: Unidade de Terapia Intensiva Pediátrica, e acolhimento familiar na UTI.

Os critérios de inclusão definidos para a seleção dos artigos foram: artigos inteiramente disponíveis relacionados ao objeto de pesquisa, artigos em idiomas português, que fossem indexados nas bases de dados. No contexto de familiares de pacientes internados em uma UTIP, visando as dores, e as dificuldades dessas famílias, artigos publicados no período de 2018 a 2021. Foram excluídas teses, dissertações e monografias. 
Unidade de Terapia Intensiva-Pediátrica: Compreensão das Necessidades dos Familiares dos Pacientes Internados

Para a extração dos dados foi utilizado um instrumento de coleta contendo: a) identificação do artigo (título, periódico, base de dados, autores, idioma e ano de publicação); b) descritores: unidade de terapia intensiva pediátrica e acolhimento familiar na UTI; c) idioma: português; d) área: enfermagem; c) abordagem: qualitativa; e) avaliação do rigor metodológico. Após a busca na base de dados foram elaboradas tabelas, visando permitir a avaliação sistemática das informações coletadas e a realização de discussões e análises.

\section{Quadro 1- Fluxograma mostrando a seleção dos artigos incluídos para o estudo}

\begin{tabular}{|c|c|c|}
\hline \multicolumn{3}{|c|}{ 'ROCEDIMENTO METODÓLOGICO } \\
\hline Bancos de dados & SCIELO & LILACS \\
\hline Descritores: & $\begin{array}{l}\text { Unidade de Terapia Intensiva } \\
\text { Pediátrica } \\
\text { Acolhimento familiar na } \\
\text { Unidade de Terapia Intensiva }\end{array}$ & $\begin{array}{l}\text { Unidade de Terapia Intensiva } \\
\text { Pediátrica } \\
\text { Acolhimento familiar na Unidade } \\
\text { de Terapia Intensiva }\end{array}$ \\
\hline Tipo de trabalho: Artigo & 108 & 441 \\
\hline Idioma: português & 44 & 210 \\
\hline $\begin{array}{l}\text { Ano de publicação: } \\
2018 \text { a } 2021\end{array}$ & 56 & 45 \\
\hline Abordagem: qualitativa & 11 & 6 \\
\hline Área da Enfermagem & 3 & 4 \\
\hline Amostra final & 3 & 4 \\
\hline
\end{tabular}

Assim, nesta investigação, a extração das informações léxicas e semanticamente mais importantes, tanto para o objeto quanto para os seus objetivos do estudo, serão também, dispostas em quadros, ranqueadas pela ordem de série (R). Para aumentar ainda mais o rigor dos dados obtidos utilizou-se a análise lexicográfica o que permitiu determinar quantitativamente a ocorrência de palavras com a 35 frequência absoluta, porcentual, chi2 e grau de significância ou $\mathrm{P}$ (significância) que foram obtidos a partir do processamento das respostas dos resumos dos artigos pelo software Iramuteq 7.2 (LAHLOU, 2012; RATINAUD \& MARCHAND, 2012).

A realização da análise sociolinguísticas utilizamos o Iramuteq 7.2. É um software gratuito e com fonte aberta, desenvolvido por Pierre Ratinaud (Lahlou, 2012; Ratinaud \& Marchand, 2012) e licenciado por GNU GPL (v2), que permite fazer análises estatísticas sobre 
Unidade de Terapia Intensiva-Pediátrica: Compreensão das Necessidades dos Familiares dos Pacientes Internados

corpus textuais e sobre tabelas indivíduos/palavras. Ele ancora-se no software $\mathrm{R}$ e na linguagem Python. A análise textual é um tipo específico de análise de dados, na qual tratamos de material verbal transcrito, ou seja, de textos (NASCIMENTO-SCHULZE \& CAMARGO, 2000).

\section{RESULTADOS E DISCURSSÕES}

No quadro 2 estão distribuídos os dados principais de artigo, tais como: a identificação do estudo, nome dos autores, título, ano de publicação e a revista. Foram encontrados 549 estudos, dos quais 07 abordavam o tema proposto, estavam dentro dos critérios adotados e foram selecionados para compor este estudo (Quadro1).

Ressaltamos que, de posse de todos os dados, foi realizada a leitura flutuante para busca do que estava por de trás das respostas, encontrar novas realidades através das mensagens sobre o que já sabemos ou pensamos saber sobre Unidade de Terapia Intensiva-Pediátrica: Compreensão das necessidades dos familiares dos pacientes internados. Todos os textos foram olhados, pensados e organizados a luz do referencial de Laurence Bardin (1977). Transcorrido esta etapa fizemos uma análise semântica de todo material na busca das unidades linguísticas que funcionam como material da análise de conteúdo: os significados. Trabalha com a sociolinguísticas que se movimenta da língua para as palavras, de modo a estabelecer uma maneira sistemática de relações (covariância) entre estruturas linguísticas e sociais. Vejamos os artigos selecionados:

Quadro 2 - Distribuição dos estudos selecionados por autores, título do estudo, ano de publicação e o nome da revista.

\begin{tabular}{|c|c|c|c|c|}
\hline Artigo & Autores & Título & Ano & Revista \\
\hline A1 & $\begin{array}{c}\text { Lima LG et.al Smeha } \\
\text { LN et.al }\end{array}$ & $\begin{array}{l}\text { A experiência da maternidade diante da } \\
\text { internação do bebê em UTI: Uma } \\
\text { montanha russa de sentimentos }\end{array}$ & 2019 & $\begin{array}{l}\text { Psicologia em } \\
\text { Estudo. }\end{array}$ \\
\hline A2 & $\begin{array}{l}\text { Ferreira MJM et.al } \\
\text { Dodt RCM et.al } \\
\text { Lima AM et.al } \\
\text { Marques DRF et.al } \\
\text { Pinheiro SMPR et.al }\end{array}$ & $\begin{array}{c}\text { Percepção dos acompanhantes sobre } \\
\text { dispositivos invasivos em uma unidade } \\
\text { de terapia intensiva pediátrica }\end{array}$ & 2018 & Enfermagem Foco \\
\hline A3 & $\begin{array}{l}\text { Souza AS et.al } \\
\text { Cabeça LPF et.al } \\
\text { Souza MA et.al } \\
\text { Melo LL et.al }\end{array}$ & $\begin{array}{l}\text { Experiências maternas diante da } \\
\text { transferência do filho para a unidade de } \\
\text { terapia intensiva }\end{array}$ & 2018 & $\begin{array}{c}\text { Revista Baiana de } \\
\text { enfermagem }\end{array}$ \\
\hline A4 & $\begin{array}{c}\text { Silva CC et.al } \\
\text { Souza MA et.al } \\
\text { Cabeça LPF et.al Melo } \\
\text { LL et.al }\end{array}$ & $\begin{array}{l}\text { Modos de ser de profissionais de } \\
\text { enfermagem em terapia intensiva } \\
\text { pediátrica: Vivências com famílias }\end{array}$ & 2020 & $\begin{array}{l}\text { Revista Mineira de } \\
\text { Enfermagem }\end{array}$ \\
\hline
\end{tabular}


Unidade de Terapia Intensiva-Pediátrica: Compreensão das Necessidades dos Familiares dos Pacientes Internados

\begin{tabular}{|c|c|c|c|c|}
\hline A5 & $\begin{array}{c}\text { Morais ES et.al } \\
\text { Castillo AMCM et.al }\end{array}$ & $\begin{array}{c}\text { A experiência dos avós de crianças } \\
\text { hospitalizadas em unidade de terapia } \\
\text { intensiva pediátrica }\end{array}$ & 2018 & $\begin{array}{c}\text { Revista da Escola } \\
\text { de Enfermagem } \\
\text { USP }\end{array}$ \\
\hline A6 & $\begin{array}{c}\text { Perão OF et.al } \\
\text { Nascimento ERP et.al } \\
\text { Padilha MICS et.al } \\
\text { Lazzari DD et.al } \\
\text { Hermida PMV et.al } \\
\text { Kersten MAC et.al }\end{array}$ & $\begin{array}{c}\text { Representações sociais de conforto para } \\
\text { familiares de pacientes em cuidados } \\
\text { paliativos na terapia intensiva }\end{array}$ & 2021 & $\begin{array}{c}\text { Revista Gaúcha de } \\
\text { Enfermagem }\end{array}$ \\
\hline A7 & $\begin{array}{c}\text { Souza PSN et.al } \\
\text { Conceição AOF et.al }\end{array}$ & $\begin{array}{c}\text { Processo de morrer em unidade de } \\
\text { terapia intensiva pediátrica }\end{array}$ & 2018 & Revista Bioética \\
\hline
\end{tabular}

Fonte: Esquematizado pelos autores

O Iramuteq 7.2 é capaz de associar diretamente os textos do banco de dados com as variáveis pré-determinadas pelo seu usuário, assim, é possível analisar a produção textual em função dessas variáveis. Esta análise configura-se análise de contrastes em que o corpus é dividido em função da variável escolhida pelo usuário. É possível comparar a produção textual entre textos em relação a determinado tema, o que foi realizado a partir do resumo dos 7 artigos selecionado. Vejamos:

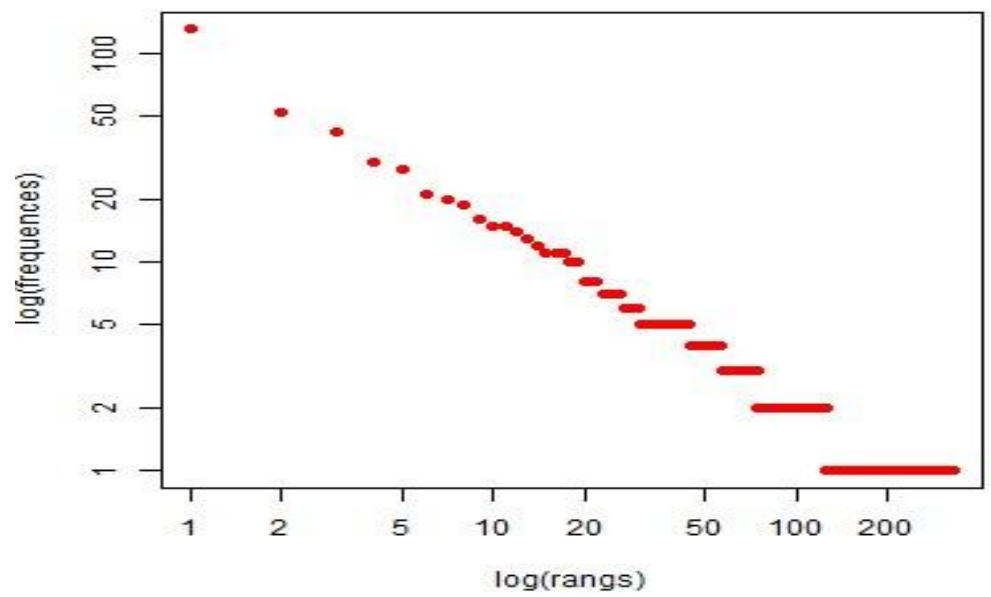

Figura 1- Diagrama de Zipf

Fonte: IRAMUTEQ (2021)

Para análise do log de frequência foram analisados os 7 (sete) artigos, com o total de 1036 ocorrências, 330 números de formas e 205 números, hápax (19,79\% de ocorrências $62,12 \%$ de formas) com média de ocorrência por texto foi de 148.00 . 
Unidade de Terapia Intensiva-Pediátrica: Compreensão das Necessidades dos Familiares dos Pacientes Internados

\begin{tabular}{|c|c|c|c|c|c|c|c|}
\hline formes & *resu... \ & *resumo2 & ${ }^{\star}$ resumo3 & *resumo4 & ${ }^{\star}$ resumo5 & *resumo6 & ${ }^{*}$ resumo 7 \\
\hline pediátrico & 0.4395 & 0.5128 & -0.2797 & 0.2939 & 0.2818 & -1.2815 & 0.378 \\
\hline terapia & 0.3059 & 0.3691 & 0.4061 & -0.3537 & -0.1899 & 0.4061 & -0.8817 \\
\hline intensivo & 0.2796 & 0.3403 & 0.3592 & -0.4209 & -0.2142 & -0.2497 & 0.2304 \\
\hline unidade & 0.2556 & 0.3139 & 0.6036 & -0.279 & -0.2397 & -0.5734 & 0.2086 \\
\hline como & 0.2235 & -0.3497 & 0.2807 & -0.8913 & -0.1592 & 0.6622 & 0.6347 \\
\hline cuidado & -0.4373 & -0.3866 & -0.9187 & -0.2576 & -0.1884 & 1.7428 & 0.5699 \\
\hline familia & -0.4795 & -0.424 & -0.4356 & 2.1498 & 0.4018 & -0.4356 & -0.5357 \\
\hline
\end{tabular}

Figura 2- Formas (palavras) evidenciadas nos resumos por frequências

Fonte: IRAMUTEQ (2021)

As formas encontradas foram: pediátrico, terapia, intensivo, unidade, como, cuidado, família. Contudo, essas formas foram possíveis para prosseguirmos, a análise de Classificação Hierárquica Descendente (CHD), o qual possibilita três escolhas diferentes junto ao Iramuteq; e optamos pela Simple SUR Textes - que realiza a análise considerando os textos sem dividilos em segmentos de texto, recomendado para respostas mais curtas, utilizado neste estudo.

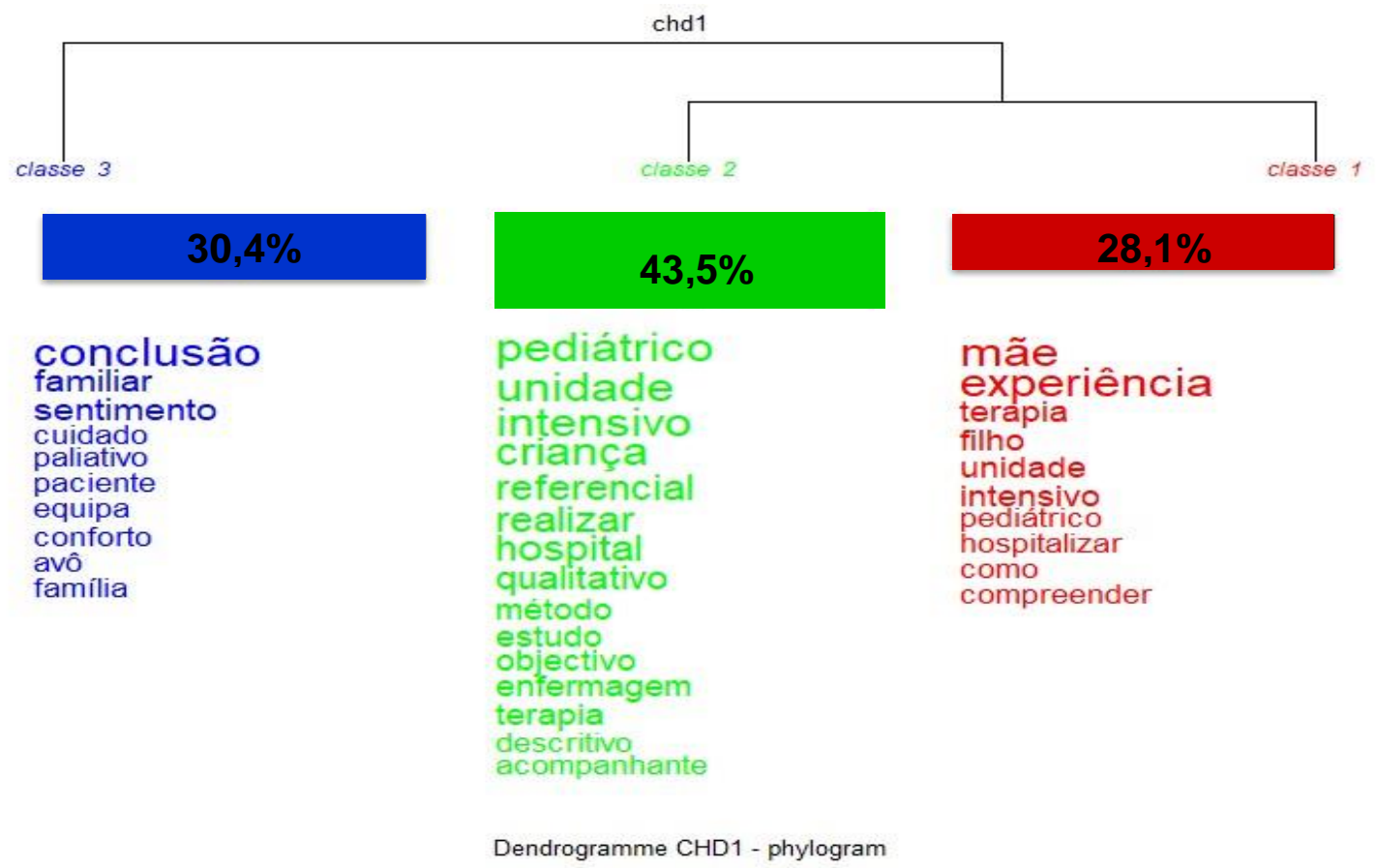

Figura 2- Dendograma I CHD

Fonte: IRAMUTEQ (2021)

$\mathrm{Na}$ aba CHD1 dos resultados, é possível ter acesso ao dendograma, que apresenta as partições que foram feitas no corpus até que se chegasse às classes finais. Lê-se o dendograma da esquerda para a direita.", utilizado aqui como exemplo, foi dividido ( $1^{\text {a }}$ partição ou iteração) em dois sub-corpus. Num segundo momento um sub-corpus foi dividido em dois ( $2^{\mathrm{a}}$ partição 
Unidade de Terapia Intensiva-Pediátrica: Compreensão das Necessidades dos Familiares dos Pacientes Internados

ou iteração), assim obteve-se a classe 3. E num terceiro momento, há mais partições, originando de um lado, as classes 1; e 2. A CHD parou aqui, pois as 3 classes mostraram-se estáveis, ou seja, compostas de unidades de segmentos de texto com vocabulário semelhante.

O dendrograma I mostra que na 1 classe as palavras que mais se evidenciaram em 28,1\% foram "Mãe" e "Experiência". Na 2 classe apresenta em 43,5\%, as palavras "Pediátrico", "Unidade", "Intensivo", "criança", "referencial", "Realizar" e "Hospital". E finalmente na terceira e última classe retratam em 30,4\%, as palavras "Conclusão", "Famíliar" e "Sentimento". Por fim, a análise findou-se no CHD apresentando as nuvens de palavras. Conforme a figura 4 a seguir:

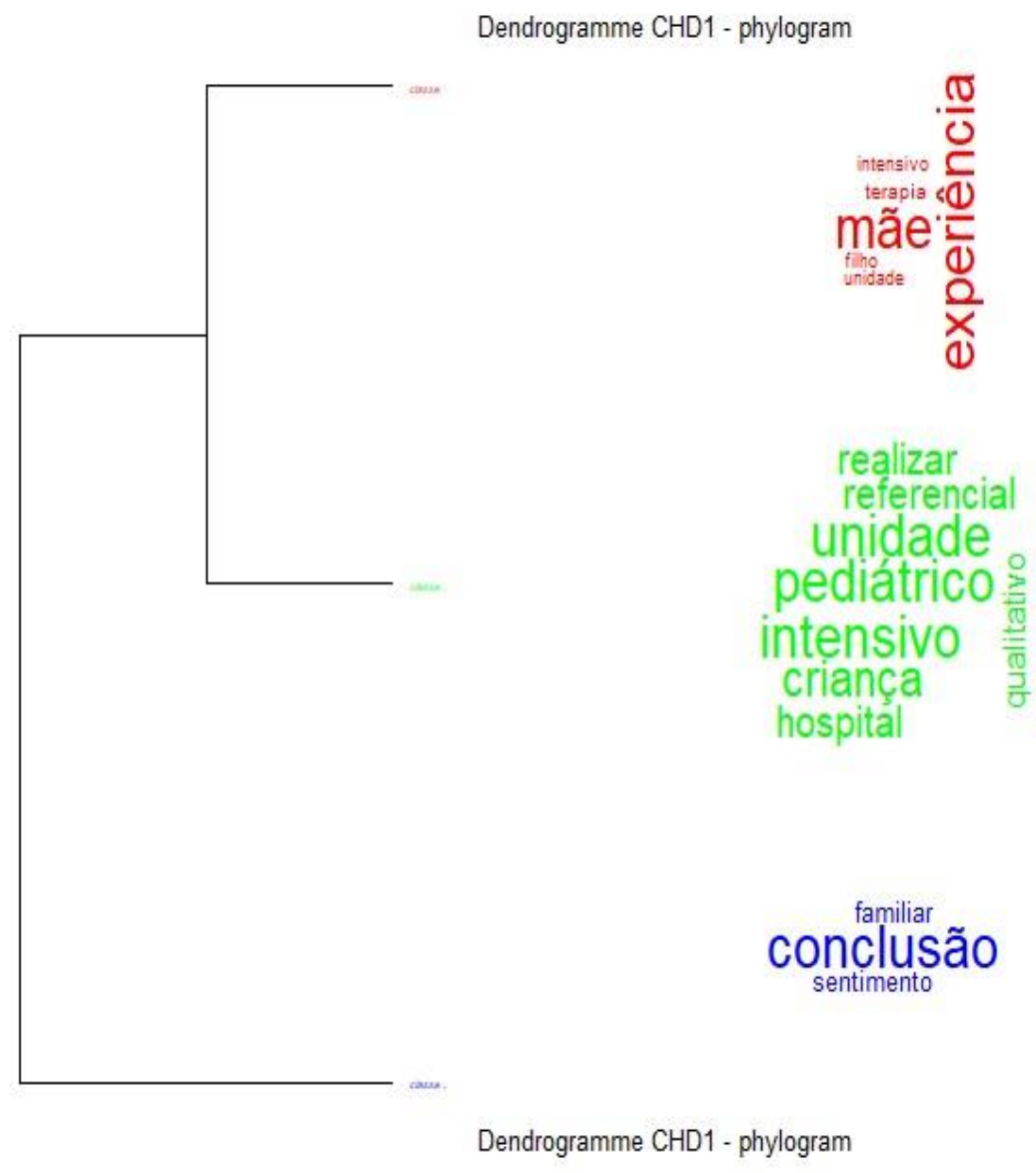

Figura 4- Dendograma I CHD

Fonte: IRAMUTEQ (2021)

A posição do CHD1 foi desenvolvida e permitiu a produção de registros, gerou palavras de registro por classe 1: Mãe, experiência; classe 2 Unidade pediátrico intensivo, criança e finalmente a classe 3: conclusão, familiar e sentimento, destacou-se duas categorias percebidas 
Unidade de Terapia Intensiva-Pediátrica: Compreensão das Necessidades dos Familiares dos Pacientes Internados

pelos pesquisadores; mostrada a seguir: a emergência de sentimentos e movimentos de comunicação captadas na linguagem verbal e não verbal.

Evidenciou-se que os estudos descritos acima, são direcionados para os familiares dos pacientes internados na UTIP. Ressaltando a importância da família no acompanhamento nos processos de cuidados nós pacientes de UTIP.

De acordo com Lima LG et. al (2019), ressalta que "a experiência materna diante da internação do bebê em UTI: Uma montanha russa de sentimentos" As hospitalizações aconteceram em seis diferentes UTIs, pediátricas e Neonatais, localizadas em cinco cidades do Estado do Rio Grande do Sul. As idades dos bebês variaram entre 0 a 38 dias, e os bebês foram referidos pelos números, de 1 a 9 , de acordo com o número que corresponde à sua mãe e as características dos bebês e das hospitalizações. O estudo destacou-se a dor emocional que é causada na mãe, acontece uma mudança muito grande na rotina familiar, isso faz com que a situação piore, pois, a mãe sente como se estivesse perdendo o controle de tudo. Com isso surge preocupação, ansiedade e insegurança.

Segundo Ferreira et.al (2018) participaram do estudo dezessete acompanhantes de pacientes internados em UTIP nos meses de julho e agosto de 2016. Desse total, 59\% eram do gênero feminino e $41 \%$ do gênero masculino. A faixa etária preponderante dos acompanhantes, neste período variou entre 20 e 29 anos, totalizando 47\%, e caracterizava-se por baixa escolaridade, pois $35 \%$ possuíam ensino fundamental completo e $23 \%$ ensino fundamental incompleto. A parte mais difícil da hospitalização de um ente querido é a introdução desses aparelhos que na maioria das vezes os familiares não têm conhecimento da função desses aparelhos e porque foi colocado, logo associa a piora do paciente a morte. O medo do desconhecido pode gerar ansiedade e angústia, por isso o cuidado de enfermagem não deve ser somente ao paciente, e sim estendido ao acompanhante/família, para que se sinta acolhido, podendo expressar seus medos e dúvidas.

O estudo de Souza et.al (2018) "experiências maternas diante da transferência do filho para a unidade de terapia intensiva" foi um serviço de Enfermagem Pediátrica (UIP) e a UTIP de um hospital de ensino, terciário, público, localizado no interior do estado de São Paulo, Brasil. O critério de inclusão dos participantes foi ser mãe que presenciou a transferência do filho da UIP para a UTIP. Foram convidadas 17 mães e 13 aceitaram participar do estudo. Os profissionais de enfermagem contam de sua vivência com as famílias trazendo muitos benefícios para o filho hospitalizado considerando-a como um elo entre a criança e a equipe. 
Unidade de Terapia Intensiva-Pediátrica: Compreensão das Necessidades dos Familiares dos Pacientes Internados

Ter a família por perto traz calma para a criança fazendo com que ela chore menos e fique mais tranquila, e torna mais fácil dos profissionais lidar com ela.

A literatura de Silva et.al (2018) sobre "Modos de ser de profissionais de enfermagem em terapia intensiva pediátrica: Vivências com famílias" O cenário da pesquisa foi a UTIP de um hospital de ensino público, no interior do estado de São Paulo, Brasil. Os participantes foram 19 profissionais de Enfermagem, sendo 10 técnicos de enfermagem e 9 enfermeiros, de ambos os sexos, com idades de 26 e 58 anos. O tempo de formação desses profissionais variou entre cinco e 36 anos, com atuação média na UTIP estudada de oito anos.

De acordo com os profissionais o impacto das notícias difíceis como a de ter um filho internado em uma UTIP não se limita ao momento da comunicação, ou seja, a família não sente o impacto somente quando está recebendo a notícia, pode durar por semanas, meses e até anos após o anúncio. Deve-se observar bem como a família recebe a notícia, como está enfrentando, e prestar o auxílio necessário para ajudar na superação da família, pois cada pessoa reage de um modo diferente, cada mãe recebe a notícia de forma singular podendo ter diversas reações como chorar, gritar, algumas reações podem ser piores como entrar em colapso, choque e até pânico.

Delineando sobre o "Unidade de terapia intensiva pediátrica" foi abordado em cinco artigos com estudos que foram realizados em: Ferreira et.al (2018), em um hospital de referência em emergência traumatoortopédico de Fortaleza. Souza et.al (2018), em um hospital de ensino, terciário, público do interior de São Paulo. Silva et.al (2020), realizado em um hospital público do interior de São Paulo. Morais et.al (2018), em um hospital público da cidade de São Paulo. Souza et.al (2018), as contribuições desse artigo foram realizadas no hospital de grande porte de Curitiba, Paraná.

Sendo que, um deles abordou a visão do profissional enfermeiro diante da família, é importante frisar que a importância do profissional no acompanhamento familiar e acolhimento deles na UTIP (A4). O artigo (A1) é uma abordagem especifica da experiência da mãe com o seu bebê na UTIP, não tirando o foco do trabalho, é importantíssimo ressalvar sobre essas questões de unidade de terapia intensiva (UTIP) no texto diz: a experiência da maternidade diante da internação do bebê em UTI: uma montanha russa de sentimentos.

Sobre a "a experiência dos avós de crianças hospitalizadas em unidade de terapia intensiva pediátrica" de Morais et.al (2018) para os avós ter a experiência de ter um neto hospitalizado em uma UTIP causa dor e sofrimento, pois para eles ter um neto internado 
Unidade de Terapia Intensiva-Pediátrica: Compreensão das Necessidades dos Familiares dos Pacientes Internados

significa a "morte", pois eles enxergam a UTIP como um lugar para morrer, onde não há mais recursos a se fazer.

E o estudo de Perdão et.al (2021) o cenário de pesquisa foi uma UTI de uma instituição hospitalar localizada em Florianópolis- Santa Catarina. A coleta de dados ocorreu no período entre julho de 2016 e dezembro de 2017, por meio de entrevistas individuais semiestruturadas, guiada por um roteiro com questões sobre o significado de UTI para o familiar, como ele se sentia ao visitar o parente na UTI, de que forma queria ser atendido, e o que pensava sobre cuidados paliativos.

Na pesquisa de Souza et.al (2018) "Processo de morrer em unidade de terapia intensiva pediátrica". Utilizou-se pesquisa qualitativa exploratório-descritiva, envolvendo a participação de trinta profissionais de enfermagem, por meio de entrevista gravada e posteriormente transcrita, coletada em quatro UTIP de hospital de grande porte de Curitiba, Paraná, Brasil. Entrevistaram-se 20 técnicos de enfermagem e 10 enfermeiros, com somente um participante do sexo masculino. A população da amostra ainda se caracteriza por $67 \%$ dos entrevistados de faixa etária entre 20 e 30 anos, $20 \%$ com idade entre 31 e 40 anos e $13 \%$ entre 41 e 50 anos. O estudo evidenciou que, para os profissionais de enfermagem, o maior tempo de contato com o paciente pediátrico, o acompanhamento do desenvolvimento da criança e a convivência com a família acabam resultando em maior dificuldade para aceitar a morte. Porém a o acolhimento e acompanhamento da equipe nesse momento é de extrema importância.

Enfim, as práticas de acolhimento a família e os cuidados paliativos oferecidos pela equipe de enfermagem tem o papel de buscar conforto para a família, e vimos que essa estratégia colhe resultados satisfatórios nós atendimento a família do paciente. Adquirir ferramentas de apoio emocional para a família na UTIP capacita a equipa de enfermagem a oferecer o melhor atendimento.

\section{CONSIDERAÇÕES FINAIS}

Consideramos ter alcançado o objetivo proposto pela pesquisa, foram destacados os aspectos relacionados ao acolhimento a família do paciente internado da UTIP, o olhar da equipe de enfermagem para com o acompanhante sobre os sentimentos apresentados pela comunicação verbal e não-verbal como por exemplo a dor e sofrimento e as dúvidas que aparecem na dinâmica do processo da internação. 
Unidade de Terapia Intensiva-Pediátrica: Compreensão das Necessidades dos Familiares dos Pacientes Internados

Ressaltamos e sugerimos a partir da pesquisa, uma perspectiva cuidadora encontro onde os profissionais são convidados, a refletir, a dialogar e a identificar os aspectos que determinam tais necessidades do contexto da internação nos serviços de saúde. A criança e a família como produtores de experiências, sentimentos, vivências e significados; acreditamos que ajudaria na melhora e recuperação do paciente, e na família a relação de confiança entre ambos, no binômio equipe $\mathrm{X}$ família/paciente.

\section{REFERÊNCIA}

AMARAL, Lídia; CALEGARI, Tatiany. Humanização da assistência de enfermagem à família na unidade de terapia intensiva pediátrica. Cogitare Enferm. p. 02, 2016.

BARBOSA, Elizabeth; RODRIGUES, Benedita. Humanização nas relações com a família: um desafio para a enfermagem em UTI pediátrica. Acta Scientiriarum. Health Sciences, vol. 26 n. $01,2004$.

BARDIN, L. Análise de conteúdo. EDIÇÃO 70. Presses Univcrsitaires de France. 1977.

BOUSSO, RS.; ANGELO, M. Buscando preservar a integridade da unidade familiar: a família vívendo a experiência de ter um filho na UTI. Rev Esc Enf USP, v. 35, n. 2, p. 172-9, jun. 2001.

BRASIL. Programa de Humanização da Assistência Hospitalar, Secretaria de Assistência à Saúde. Programas e relatórios. n. 20. Brasília (DF): MS; 2001

CARDOSO, Priscila Fernandes Gonçalves. Ética e projetos profissionais: os diferentes caminhos do Serviço Social no Brasil. Campinas, SP: Papel Social, 2013.

COSTA, R. A responsabilidade do enfermeiro na humanização da assistência em terapia intensiva neonatal. Rev Enferm UNISA, n.2 p. 44-4, 2001.

DOISE, W.; CLEMENCE, A.; LORENZI-CIOLDI, F. Représentations sociales et analyses de données. Grenoble: P.U.G. 1992.

FERREIRA. M.J.M.; DODT. M.C.R.; LIMA. M.A.; MARQUES. F.D.; PINHEIRO. E.P.M. Percepção dos acompanhantes sobre dispositivos invasivos em uma unidade de terapia Intensiva pediátrica. Enfermagem em foco. 2018.9 (2): 18-22. Disponível em: https://fiadmin.bvsalud.org/document. 
Unidade de Terapia Intensiva-Pediátrica: Compreensão das Necessidades dos Familiares dos Pacientes Internados

FREIRE. M.A.L.; STORINO. O.L.; HORTA. C.N.; MAGALHÃES. P.R.; LIMA. T. O acolhimento sob a ótica de profissionais da equipe de saúde da família. Rev. Min. Enferm.;12(2): 271-277, abr./jun., 2008

LAHLOU, S. Text Mining Methods: An answer to Chartier and Meunier. Papers on Social Representations, 20 (38), 1.-7.,2012.

LEBART, L.; SALEM, A. Statistique textuelle. Paris: DUNOP. 1994.

LIMA.G. L.; SMEHA.L.N. A experiência da maternidade diante da internação do bebê em UTI: Uma montanha russa de sentimentos. Psicologia em Estudo. v. 24, e38179, 2019. Disponível em: Doi: 10.4025/psicolestud. v24i0.38179.

MOLINA, Rosemeire; BERCINI, Luciana; VARELA, Patrícia; MARCON, Sonia; CASTILHO, Sonia. Presença da família nas unidades de terapia intensiva pediátrica e neonatal: visão da equipe multidisciplinar. Esc. Anna Nery R. Enferm, p. 438, 2007.

MOLINA, Rosemeire; FONSECA, Eliet; WAIDMAN, Maria; MARCON, Sonia. A percepção da família sobre sua presença em Unidade de Terapia Intensiva Pediátrica e Neonatal.

VER.ESC, ENFERM.USP vol.43 no.3 São https://doi.org/10.1590/S008062342009000300019

MORAIS. E.S.; MENDES-CASTILLO. A.M.C. A experiência dos avós de crianças hospitalizadas em unidade de terapia intensiva pediátrica. Rev Esc Enferm. USP.2018;52:e03395. DOI:http://dx.doi.org/10.1590/S1980220X2017040003395

NASCIMENTO-SCHULZE, C. M.; CAMARGO, B. V. Psicologia social, representações sociais e métodos. Temas de psicologia. Ribeirão Preto, 8 (3), 287-299, 2000.

NOGARE, Diego. Apogeu do humanismo: humanismo marxista, existencialista e cristão. In: OLIVEIRA, Maria. et al. A melodia da humanização: reflexão sobre o cuidado no al. A melodia da humanização: reflexão sobre o cuidado, p. 22 - 29, 2001

PEDROSO, REG.; BOUSSO. SR.Ciência, Cuidado e Saúde Maringá, v. 2, n. 2, p. 123-129, jul./dez. 2003.

PERÃO. O.F.; NASCIMENTO. E.R.P.; PADILHA. M.I.C.S.; LAZZARI. D.D.; HERMIDA. P.M.V.; KERSTEN. M.A.C. Representações sociais de conforto para familiares de pacientes em cuidados paliativos na terapia intensiva. Rev Gaúcha Enferm. 2021;42:e20190434. doi: https://doi.org/10.1590/1983-1447.2021.20190434.

PESSALOCIA, Juliana; SILVA, Larriny; JESUS, Lailane; SILVEIRA, Renata; OTONI, Alba. Atuação da equipe de enfermagem em UTI pediátrica: um enfoque na humanização. Ver. Enferm. Cent. O. Min. 2012.

PÊGO, Carina; BARROS, Marcela. Unidade de Terapia Intensiva Pediátrica: expectativas e sentimentos dos Pais da Criança Gravemente Enferma. Rev. Brasileira de Ciências da Saúde vol.21 no.1 2017. 
Unidade de Terapia Intensiva-Pediátrica: Compreensão das Necessidades dos Familiares dos Pacientes Internados

RODRIGUES, Amanda; CALEGORI, Tatiany. Humanização da assistência da unidade de terapia intensiva pediátrica: perspectiva da equipe de enfermagem. Min Enferm. 2016.

RATINAUD, P., \& MARCHAND, P. Application de la méthode ALCESTE à de "gros" corpus et stabilité des "mondes lexicaux": analyse du "CableGate" avec IraMuTeQ. Em: Actes des 11eme Journées internationales d'Analyse statistique des Données Textuelles (835-844). Presented at the 11eme Journées internationales d'Analyse statistique des Données Textuelles. JADT. Liège, 2012.

RAAD, A.J. A realidade das mães numa unidade de terapia intensiva neonatal. Revista de Psicologia da Vetor. Editora, São Paulo, v. 7, n.2, p. 85-92, jul-dez. 2006.

SILVA. C.C.; SOUZA. M.A.; CABEÇA. L.P.F.; MELO. L.L. Modos de ser de profissionais de Enfermagem em terapia intensiva pediátrica: vivências com famílias. REME - Rev Min Enferm. 2020;24:e-1305. Disponível em: DOI: 10.5935/1415-2762.20200042.

SOUZA. A. S.; CABEÇA. L.P.F.; SOUZA. M.A.; MELO. L.L. Experiências maternas diante da transferência do filho para a unidade de terapia intensiva. Rev baiana enferm. 2018;32:e25160. Disponível em: DOI 10.18471/rbe. v32.2516.

SOUZA. N.S.P.; CONCEIÇÃO. F.O.A. Processo de morrer em unidade de terapia intensiva pediátrica. Revista bioética. vol.26 no.1 Brasília Jan./mar. 2018. Disponível em: http://dx.doi.org/10.1590/1983-80422018261234.

SOUSA, Francisca; SANTOS, Danilo; LIMA, Heloísa; SILVA, Dennyse; CABEÇA, Luciana; PERDIGÃO, Ericka. O familiar na unidade de terapia intensiva pediátrica: um contexto revelador de necessidades. Cuidado é fundamental. Vol.7, dez, 2014.

SOUSA. M.; GEORGINA. F.; SANTOS. A.; MARCELO. D.; LIMA. O.F.; ROSÁRIO. H.; SILVA. M.; CRISTINA. D.; CABEÇA. F.P.; LUCIANA; PERDIGÃO. L.; LETÍCIA. E. O familiar na unidade de terapia intensiva pediátrica: um contexto revelador de necessidades. Revista de Pesquisa Cuidado é Fundamental Online, vol. 7, diciembre, 2015, pp. 77-94.

TRAVELBEE, J. Intervención en enfermería psiquiátrica. Cali: Carvajal, 1979. 
Unidade de Terapia Intensiva-Pediátrica: Compreensão das Necessidades dos Familiares dos

Pacientes Internados

\begin{abstract}
The hospitalization of a patient in the Pediatric Intensive Care Unit (ICU-P), usually occurs in a acute and severe state, leaving little time for family members to adjust and understand the sociopathological factors. Faced with this situation, the Family members may feel helpless and find it difficult to mobilize, causing diferente types of needs to emerge that can be disaggregated by the lack of information and prior knowledge about the ICU environment. Objective: To investigate the challengs of family members of patients who are hosptalized in the Pediatric Intensive Care Unit (ICU-P). Method: This is a literature review that seeks to understand the difficulties of Family members of patients admitted to ICU-P, and understand how the host Family are essential. The work ocurred through a literary research that included researches carried out based on scientific articles, available in the databases: Scielo and Lilacs, during the year 2018 to 2021. Result: demonstrate how important it is to understand and show sympathy to the family of the hospitalized patient in the ICU-P, and the effectiveness of having a structural and positive team When seeing a family as a whole in the bio-psyco-social process of treating the child who is affected in the ICU-P.
\end{abstract}

Key Word: Reception, Pediatric Intensive Care Unit, family.

\title{
Resumem
}

La hospitalización de un paciente en la Unidad de Cuidados Intensivos Pediátricos (UCI-P), suele ocurrir en un estado agudo y severo, dejando poco tiempo para que los familiares se ajusten y comprendan los factores sociopatológicos. Ante esta situación, los familiares pueden sentirse desamparados y tener dificultades para movilizarse, provocando que surjan diferentes tipos de necesidades que pueden desagregarse por la falta de información y conocimiento previo sobre el entorno de la UCI. Objetivo: Investigar los desafíos de los familiares de los pacientes hospitalizados en la Unidad de Cuidados Intensivos Pediátricos (UCI-P). Método: Se trata de una revisión de la literatura que busca comprender las dificultades de los familiares de los pacientes ingresados en UCI-P, y comprender cómo la Familia de acogida es fundamental. El trabajo se dio a través de una investigación literaria que incluyó investigaciones realizadas con base en artículos científicos, disponibles en las bases de datos: Scielo y Lilacs, durante el año 2018 al 2021. Resultado: demostrar lo importante que es comprender y mostrar simpatía a la familia del paciente hospitalizado en la UCI-P, y la efectividad de contar con un equipo estructural y positivo al ver a la familia en su conjunto en el proceso bio-psicosocial de atender al niño afectado en la UCI-P.

Palabra clave: Recepción, Unidad de Cuidados Intensivos Pediátricos, familia. 